\title{
Interventional Radiology Evolution: Our Past Guides us to Our Future
}

It is a great privilege to be the keynote speaker for the 2019 Pan Arab Interventional Radiology Society (PAIRS) meeting. Thank you for this honor.

I am going to discuss the parallel evolution of PAIRS and Society of Interventional Radiology (SIR) and highlight similarities between the two Societies.

Over 60 years ago, Charles Dotter was tinkering with endovascular tools. As he realized catheters could be used for more than diagnostic tools, our specialty was born. Following Dotter, number of innovative radiologists began to widen the scope of catheter-based therapy. This early period of imagination and development was occurring in the United States, Europe, and Asia. In a short period, these innovative physicians began to aggregate at meetings, and interventional radiology (IR) began to evolve as a specialty.

Early on, there was no formal training, but there was a fertile substrate for the growth and development of our specialty. As imaging improved with advances such as digital subtraction angiography and cross-sectional imaging, the ability to perform catheter-based interventions was greatly facilitated. It soon became obvious that these "special procedures" could replace surgical procedures, and less invasive therapy could manage many medical problems. The innovative minds of these founders were the catalyst for the evolution of the specialty.

Now in 2019, after 50 years, IR is a mature specialty in the US and in other regions of the world. SIR was formed in 1973 and had its' first annual meeting in 1975. A mandatory and critical step in the development of a scientific society is having the society's scientific accomplishments documented and recognized as valued science. This happens with the creation of a scientific journal. The first edition of the Journal of Vascular and Interventional Radiology was printed in 1990. At the same time, fellowships were being created at all of the major IR centers in the US. Initially, unaccredited but eventually the programs developed an accreditation process insuring standardization and quality for trainees. Finally, in 2012, the American Board of Medical Specialties recognized IR as its own specialty: A 40 years' process.

At this point, let's introduce the concept of parallel evolution. Parallel evolution is the independent evolution of similar traits arising in like-species that had similar ancestral conditions. The more closely related the lineages, the more similar the responses to challenges. An Illustration of this is two different fish not of the same species in different lakes in different regions of the world that have evolved and look almost identical. They are genetically similar but not identical. They have similar environmental forces, so they evolve in a similar manner, looking almost identical.

PAIRS and SIR have undergone a parallel evolution. The evolution of PAIRS is of particular interest because it has occurred in a shorter timeline than SIR. Similar to the SIR, the history began with small groups of IRs in the region meeting and discussing cases and sharing innovative concepts. It was in Cyprus in 1996 that ideas of forming a society in the region were discussed and within a decade, there the first scientific meeting occurred. Two years after the first PAIRS meeting, a session at Cardiovascular and Interventional Radiology Society of Europe (CIRSE) entitled "CIRSE meets PAIRS" occurred. This landmark session introduced PAIRS to the world outside the Middle East and established PAIRS as a scientific contributor in the global world of IR. Shortly after this, in 2014, PAIRS became an independent meeting, with a permanent meeting location, inclusively welcoming all IRs to Dubai. In 2017, shortly after the formation of the Society, the Arab Journal of Interventional Radiology (AJIR) was born giving PAIRS scientific credibility.

As we look at the parallel evolution of the SIR and PAIRS, an important observation is the diversity within PAIRS. Leaders come from multiple countries in the region, all untied with a common purpose. An equally important observation is that three of the first seven presidents of PAIRS were women. This forward-thinking achievement is unprecedented in other IR societies and in medicine in general.

The AJIR provides PAIRS with a home for research and investigation in the Arab region. The growth and development of this journal is vital to the academic and scientific recognition of PAIRS as scientific society. I have taken the time to read articles published in AJIR in the past issues, and the importance of original research is acknowledged and recognized as an area for improvement and growth. I am quite certain that in a decade when we reflect back on the progress of AJIR, dramatic increases in submissions will be noted.

The evolution of a specialty can be reflected in changes in the table of contents of the journal over time. Adaptation can be observed as new technologies develop for different types of procedures and as new procedures develop that did not previously exist. In IR, this is a rapid evolution. This is easily recognized with the evolution of interventional oncology. It is imperative that PAIRS and AJIR keep pace with this evolution.

Looking at the most recent table of contents in AJIR, parallel evolution becomes evident. Topics include 
stent placement, dialysis, biodegradable stents, and radiofrequency ablation. What is obvious to me is that there is little that is geo-specific. This table of contents could have been for any journal of IR anywhere on the globe. Looking back at previous keynote lectures from the past PAIRS meetings, there is often a common theme. This theme is of issues and challenges that are specific to the Arab region. As I reflected on my reading of articles in AJIR, I could not help but notice the many similarities both societies share. There are many more similarities than differences. Many of our opportunities and challenges are universal issues. What are the similar issues? They include epidemics of diabetes and critical limb ischemia as well as significant cost constraints in the health-care systems. We face competition from other specialists that want to do image-guided therapies. We have to become more clinical but this is difficult to do in traditional radiology practices. We have to continue to train excellent residents and fellows while being inclusive to gender and ethnicity. We must manage these issues while working long days with concerns for radiation exposure and ergonomic injuries.

Another AJIR article discusses the Egyptian female experience in IR. The author points out that there is a dearth of women interventionalists lists in Egypt, she goes on to point out that in the UK, the same problem exists and, it is even worse in the US where only somewhere between and $2 \%$ and $12 \%$ of women trainees in radiology end up in IR. In SIR, three of the founding physicians were women, but it took 30 years to have a woman president. In PAIRS, three of the first seven presidents were women. Evolution is rapid in PAIRS.

An AJIR article, "IR Practice in Saudi Arabia: Are we driving on soft Sand?" discusses the universal dilemma of IR not having a clinical face. "It is too common and alarming story that despite IR's central role in patient care, it suffers from relative anonymity. Many practitioners, residents, and medical students in Saudi Arabia and worldwide have a misconception about IR or even never heard about it." In the United States, this problem has been a major contributor to the formation of a new IR specialty separate from diagnostic radiology. The need to practice clinical medicine by IR and the need to be a high volume producer in DR has forced a divergent evolutionary path.

Having been part of SIR for 30 years, I would offer five suggestions for success of PAIRS as the young society evolves:

1. Be an inclusive society. Make room for everyone. Academicians and private practitioners from all types of groups. This inclusiveness will prevent splintering and division of the society

2. Insist on the development of a strong clinical practice. Without a clinical face, the mutual respect from clinical colleagues will not occur. This must be incorporated into your training programs at an early stage

3. Do not abandon peripheral artery disease interventions. There is often fierce competition with other specialties in this area, but IR is well suited to manage these patients and to do the complex procedures required to treat these patients. IR should be major contributors in this field

4. Take the lead in the development of standards and policies. Collaborate with other specialties to work on multisocietal guidelines and standards. Be leaders in this area

5. Continue to foster, support, and fund research and innovation. That is the lifeline for our survival. This is what allows us to survive and evolve.

In conclusion, our two Societies have much in common, and we have experienced a parallel evolution. We should work together and collaborate taking advantages of our combined strengths to overcome our mutual challenges. Looking at the rapid development and growth of PAIRS, I must congratulate of the leaders and all of the members for making this new society a global contributor in the IR community.

James F. Benenati

Department of Interventional Radiology, Miami Cardiac and Vascular Institute, Miami, Florida, USA. E-mail: jfbmobile@me.com

This is an open access journal, and articles are distributed under the terms of the Creative Commons Attribution-NonCommercial-ShareAlike 4.0 License, which allows others to remix, tweak, and build upon the work non-commercially, as long as appropriate credit is given and the new creations are licensed under the identical terms.

\begin{tabular}{|l|l|}
\hline \multicolumn{2}{|c|}{ Access this article online } \\
\hline Quick Response Code: & Website: \\
& www.arabjir.com \\
\cline { 2 - 2 } & DOI: \\
\hline
\end{tabular}

How to cite this article: Benenati JF. Interventional radiology evolution: Our past guides us to our future. Arab J Intervent Radiol 2019;3:39-40. 